\title{
The efficiency is a single criterion for choosing the type of interaxle drive
}

\author{
Andrey $V$. Efimov*, Sergey $O$. Kireev, and Marina $V$. Korchagina \\ Don State Technical University, 344000 Rostov-on-Don, Russia
}

\begin{abstract}
The efficiency is proposed as a single criterion for choosing the type of interaxle drive. Dependence of efficiency on road conditions is shown. On the basis of the proposed approach, the opportunity to select the type of interaxle drive by comparing the quantitative indices, while analyzing the entire range of road conditions, was demonstrated.
\end{abstract}

When the wheeled vehicle is moving, there are factors, often simultaneous, presenting conflicting requirements to drive of the driving wheels. First, there is almost always a certain kinematic disparity between the driving axles. In the presence of a kinematic disparity, it is required that, while maintaining a constant drive of the driving wheels, that is, maintaining the distribution of torque in a constant ratio, differential effect to take place, in other words, the wheels should have the ability to rotate at different speeds. In the absence of differential effect, the presence of a kinematic disparity leads to a circulation of parasitic power in the vehicle transmission.

Secondly, there may be different conditions of the adhesion of the wheels. When the adhesion deteriorates, the property of the differential to divide the torque in a certain ratio acquires a negative character. The impossibility, due to the reduction of adhesion, to realize a greater torque by the wheels of one axle causes a reduction in the torque applied to another axle having normal adhesion conditions. This means that the tractive force of not only the low-adhesion axle, but also the normal-adhesion axle, is reduced. In this case, in order to maintain the high tractive and economic qualities of the vehicle, it is necessary to change the former ratio between the torques, that is, their redistribution must take place. In addition to the differential and locked drive, there are a number of self-locking differentials, many of which have spread.

It should be noted a different approach of the authors to assess the expediency of using different types of drive. As pointed out in [1, p.15], [2, p.17], there is still no single point of view on creating a scheme of four-wheel drive vehicles transmission, rational from the standpoint of fuel efficiency. In most cases, the choice of the transmission scheme is based only qualitatively or by the results of testing a limited number of cars of one model; numerical criteria is needed for the energy perfection of the transmission, which would make it possible to make an objective comparison.

As an optimizing parameter, a single parameter is needed, which would be decreasing both from increased slippage and from the circulation of parasitic power. It is proposed to

\footnotetext{
* Corresponding author: efimovav2007@yandex.ru
} 
choose the efficiency, which reflects the loss for wheel slippage, as an optimization criterion. For machines with two driving axles, this coefficient equals to:

$$
\eta_{\delta}=1-\frac{N_{\delta 1}+N_{\delta 2}}{N_{K}},
$$

where $N_{K}$ - power, supplied to the driving wheels; $N_{\delta 1}, N_{\delta 2}$ - power, lost on slippage, respectively, by the wheels of the front and rear axles.

$N_{K}$ - power, supplied to the driving axles,

$$
N_{K}=T_{1} V_{T 1}+T_{2} V_{T 2},
$$

where $T_{1}, T_{2}$ - tractive forces, respectively, of the front and rear axles; $V_{T 1}, V_{T 2}-$ the circumferential (theoretical) speeds of the front and rear wheels can be expressed through their slippage:

$$
\begin{gathered}
V_{T 1}=V_{1} \frac{1}{1-\delta_{1}}, \\
V_{T 2}=V_{2} \frac{1}{1-\delta_{2}},
\end{gathered}
$$

where $V_{1}, V_{2}$ - the actual speeds of the front and rear wheels, respectively; $N_{\delta 1}$ and $N_{\delta 2}$ - the powers, lost on slippage by the first and second axles,

$$
\begin{gathered}
N_{\delta 1}=T_{1} V_{\delta 1} \\
N_{\delta 2}=T_{2} V_{\delta 2},
\end{gathered}
$$

where $V_{\delta 1}, V_{\delta 2}$ - the difference between the circumferential and actual speed of the front and rear wheels respectively,

$$
\begin{gathered}
V_{\delta 1}=V_{1} \frac{\delta_{1}}{1-\delta_{1}}, \\
V_{\delta 2}=V_{2} \frac{\delta_{2}}{1-\delta_{2}} .
\end{gathered}
$$

Thus, the efficiency of a car with two driving axles

$$
\eta_{\delta}=1-\frac{T_{1} V_{1} \frac{\delta_{1}}{1-\delta_{1}}+T_{2} V_{2} \frac{\delta_{2}}{1-\delta_{2}}}{T_{1} V_{1} \frac{1}{1-\delta_{1}}+T_{2} V_{2} \frac{1}{1-\delta_{2}}}
$$


In $[3, \mathrm{p} .120]$ it is proved that the value of the efficiency is reduced both with an increase in slippage in the case of a differential drive and with an increase in the kinematic disparity between the front and rear wheels in the case of a locked drive. Since the process of overcoming a section with reduced adhesion, as a rule, is of a short duration, and the kinematic disparity between the wheels of the front and rear axles is often not constant in magnitude, then it is necessary to consider the task using methods of dynamics, using a suitable mathematical model, for example, this [4, p.94]. The motion of the vehicle in the time interval from 0 to $\mathrm{T}$ will be considered. The length of the time interval should be selected, proceeding from the fact that after the passage of time $\mathrm{T}$, the process becomes steady. Figure 1 shows a graph of the time dependence of the efficiency of a wheeled vehicle with two different types of interaxle drive: differential and locked.

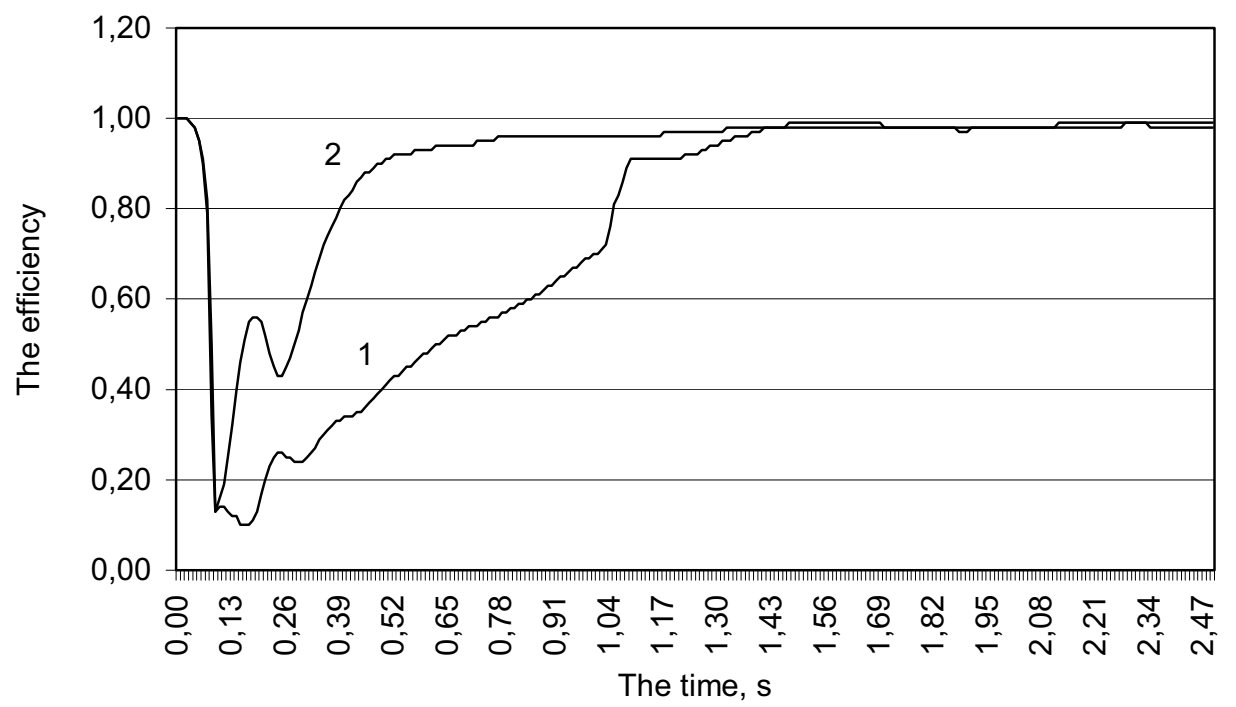

Fig. 1. Graph of the time dependence of the efficiency of the wheeled vehicle with a differential interaxial drive. Curve 1 - for a vehicle with a differential drive, curve 2 - for a vehicle with a locked drive.

The simulation has been carried out under the following conditions: the vehicle is moved from the place, there is no kinematic disparity, the coefficient of front wheels adhesion is 0.8 , and of rear wheels - is initially 0.2 , and after overcoming of $1.5 \mathrm{~m}$ distance by the vehicle, it becomes 0.8 , which means that the area with reduced adhesion is overcome.

The efficiency $\eta_{\delta}$ takes a variety of values over a period of time from 0 to $\mathrm{T}$, therefore under certain road conditions the average value of the efficiency for a period of time from 0 to $\mathrm{T}$ can be determined from the expression:

$$
\eta_{\delta}^{C P}=\int_{0}^{T} \eta_{\delta} d t .
$$

To investigate the efficiency in the entire range of road conditions, it is necessary to set a range of road conditions. As coordinates, we choose: the coefficient of adhesion of the slipping axle $\varphi_{1}$ and the kinematic disparity, $m_{H}$, where 


$$
m_{H}=\left(\frac{V^{\prime}-V^{\prime \prime}}{V^{\prime \prime}}\right) 100 \%
$$

where $V^{\prime}$ - the speed of the advancing axle, $V^{\prime \prime}$ - the speed of the lagging axle, $V^{\prime}>V^{\prime \prime}$.

After that, the entire range of road conditions will be divided by a certain step, and the movement of the vehicle will be simulated at each point of the range of road conditions, then $\eta_{\delta}^{C P}$ will be calculated for each point by formula (3) for each point of the range of road conditions.

The results obtained with the help of a mathematical model are presented in Figures 2 and 3.

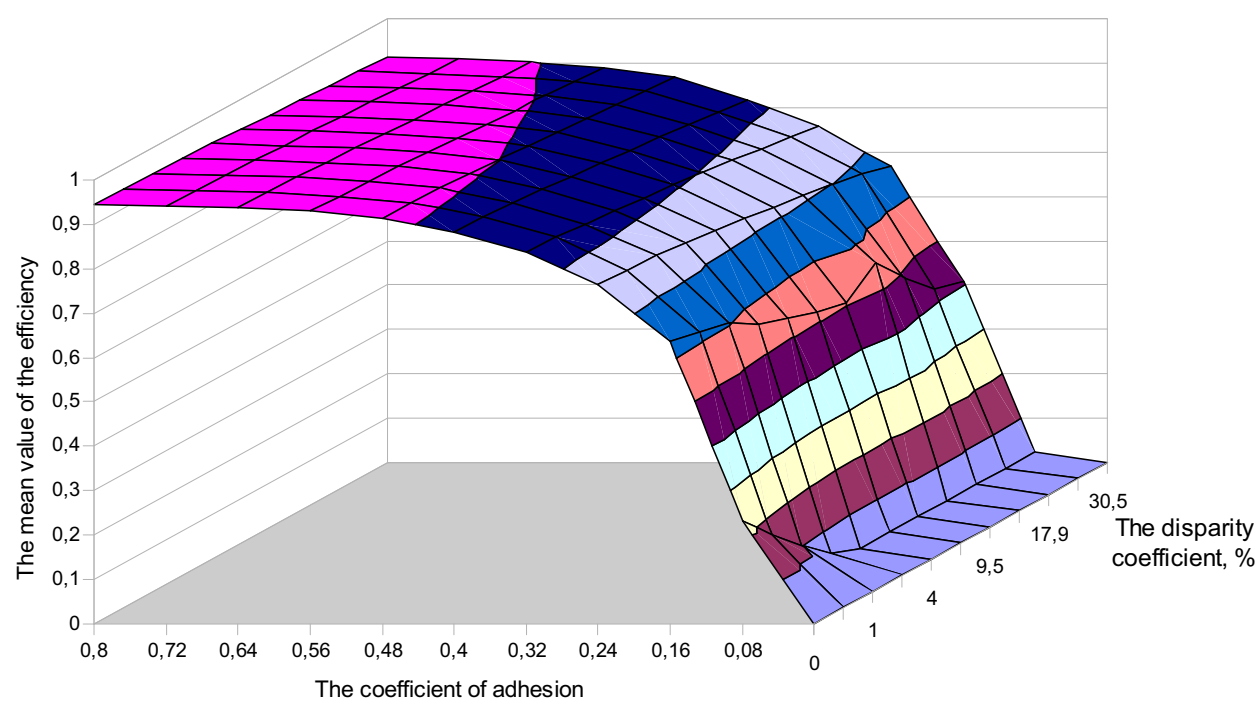

Fig. 2. Dependence of the mean value of the efficiency $\eta_{\delta}^{c p}$ on the disparity coefficient $m H$ and the coefficient of adhesion of the slipping axle $\varphi^{\prime}$ for a vehicle with a differential interaxle drive.

The obtained dependences are analyzed. When the adhesion deteriorates, the efficiency of the wheeled vehicle with the differential drive is reduced by a larger amount, in comparison with the efficiency of the wheeled vehicle equipped with a locked drive. This is due to the greater slippage of the wheels of a vehicle equipped with a differential drive, compared to a vehicle equipped with a locked drive.

The obtained dependences are analyzed. When the adhesion deteriorates, the efficiency of the wheeled vehicle with the differential drive is reduced by a larger amount, in comparison with the efficiency of the wheeled vehicle equipped with a locked drive. This is due to the greater slippage of the wheels of a vehicle equipped with a differential drive, compared to a vehicle equipped with a locked drive.

With good adhesion and the presence of a kinematic disparity, the efficiency of a wheeled vehicle equipped with a locked drive has lower values, compared to the efficiency of a vehicle equipped with a differential drive. 


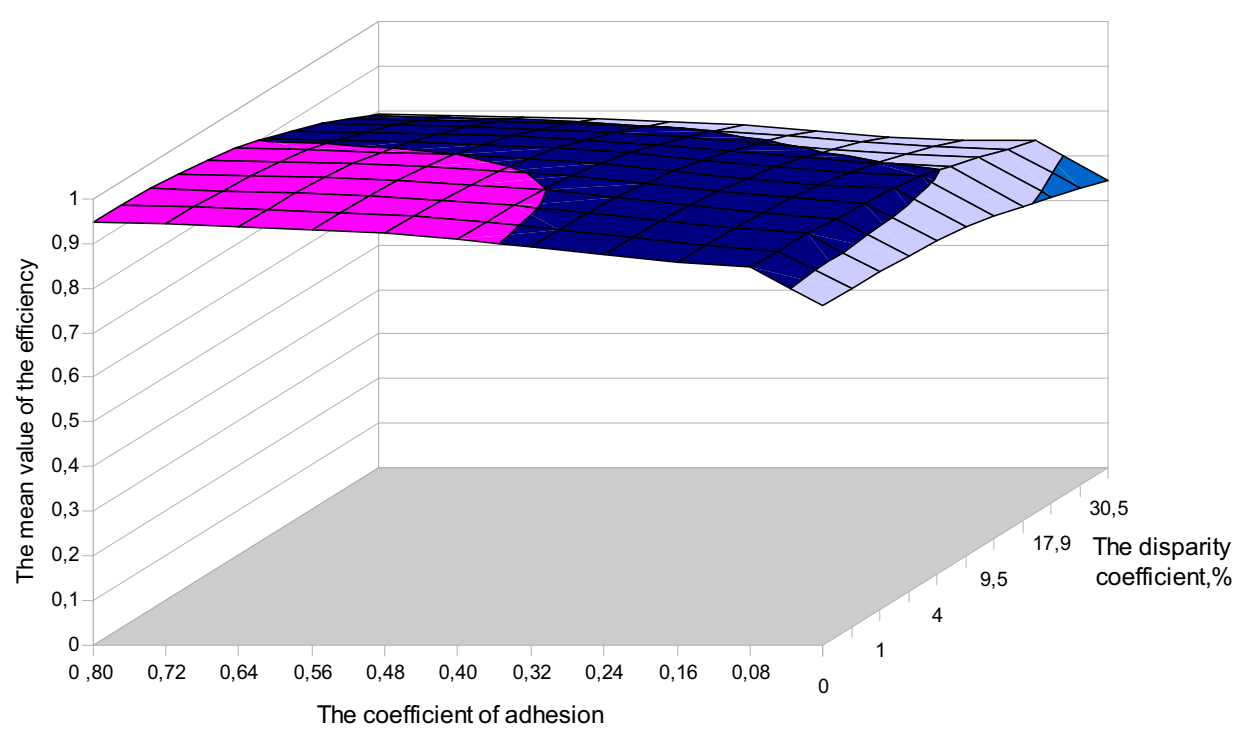

Fig. 3. Dependence of the mean value of the efficiency $\eta_{\delta}^{c p}$ on the disparity coefficient $m_{H}$ and the coefficient of adhesion of the slipping axle $\varphi^{\prime}$ for a vehicle with a locked interaxle drive.

If there is a kinematic disparity with a simultaneous deterioration in the adhesion, the efficiency the wheeled vehicle equipped with a locked interaxle drive is further reduced compared to the movement of the vehicle under the same conditions, but without a kinematic disparity. With a complete loss of adhesion by the wheels of one of the axles, a vehicle equipped with a differential interaxial drive can't get off the place, so the efficiency in this case equals to 0.

\section{Conclusions}

1. The obtained dependences correspond to the known data on the properties of the differential and locked drive, what confirms the correctness of the chosen approach.

2. The offered criterion allows to carry out a choice of type of the interaxial drive on the basis of quantitative comparison of indicators.

\section{References}

1. G.A. Smirnov, A.A. Kupreyanov, D.K. Guchkov, Automobile industry, 5, 15 (1984)

2. A.Y. Barykin, Automobile industry, 9, 17 (2004)

3. A.F. Andreev, V.V. Vantsevich, A.Kh. Lefarov, Differentials of wheel vehicles (Machine building, Moscow, 1986)

4. A.V. Efimov, S.O. Kireev, M.V. Korchagina, Problems of machine building and machine reliability, 1, 94 (2018) 